\title{
Renoprotective effect of erythropoietin via modulation of the STAT6/MAPK/NF-кB pathway in ischemia/reperfusion injury after renal transplantation
}

\author{
JINHUA ZHANG ${ }^{*}$, DAQIANG ZHAO*, NING NA* , HENG LI, BIN MIAO, \\ LIANGQING HONG and ZHENGYU HUANG \\ Department of Kidney Transplantation, The Third Affiliated Hospital of \\ Sun Yat-sen University, Guangzhou, Guangdong 510630, P.R. China
}

Received September 12, 2016; Accepted October 2, 2017

DOI: $10.3892 / \mathrm{ijmm} .2017 .3204$

\begin{abstract}
Ischemia/reperfusion injury (IRI) commonly occurs in renal transplantation. Erythropoietin (EPO) exerts a protective effect in IRI. Toinvestigate the underlying molecular mechanism, rat models of renal IRI were established and treated with EPO and/or lentivirus-mediated EPO-siRNA, the signal transducer and activator of transcription 6 (STAT6) inhibitor AS1517499, the JNK inhibitor SP600125, the p38 mitogen-activated protein kinase (MAPK) inhibitor SB203580, and the nuclear factor (NF)- $\kappa B$ inhibitor lactacystin. Histological examination revealed that EPO protected the kidney from IRI, through decreasing the extent of tissue congestion and inflammatory cell infiltration; however, EPO siRNA did not exert the same protective effect. In addition, the EPO level was inversely associated with renal IRI. EPO downregulated the expression of interferon- $\gamma$, interleukin (IL)-4, creatinine and caspase-3, and upregulated the expression of IL-10, thymic stromal lymphopoietin, STAT6, p-JNK and p-p38, while the opposite effects were observed with the administration of EPO-siRNA and the specific respective inhibitors. Further results revealed that MAPK (p-JNK and p-p38) acted upstream of NF- $\kappa B$, and that NF- $\kappa \mathrm{B}$ signaling regulated the expression of caspase-1 and -3 , which may be responsible for the cytotoxicity associated with IRI. Taken together, the results of the present study demonstrated that EPO exerted a protective effect in renal IRI via the STAT6/MAPK/NF-kB pathway. This protective effect of EPO may improve reperfusion tolerance in ischemic kidneys and benefit transplant recipients.
\end{abstract}

Correspondence to: Professor Zhengyu Huang, Department of Kidney Transplantation, The Third Affiliated Hospital of Sun Yat-sen University, 600 Tianhe Road, Guangzhou, Guangdong 510630, P.R. China

E-mail: h_zhengyu@163.com

\section{*Contributed equally}

Key words: renal transplantation, ischemia/reperfusion injury, erythropoietin, signal transducer and activator of transcription 6, mitogen-activated protein kinase, nuclear factor- $\kappa \mathrm{B}$

\section{Introduction}

Kidney transplantation is an efficacious treatment for patients with end-stage renal disease $(1,2)$. The in situ regional cooling technique with static cold storage was developed to maximize the benefit of the donated kidney for the recipient (3), and has achieved excellent kidney graft function and good graft survival (4). However, renal ischemia/reperfusion injury (IRI), which is a major cause of acute renal injury (formerly referred to as acute renal failure) and a risk factor for the quality of a kidney graft, rejection and renal fibrosis, is directly correlated with the survival of the recipient $(5,6)$. Furthermore, the complex association of pathophysiological processes with inflammation makes the ischemic kidney injury an important risk factor for progression of chronic kidney disease (7). It was documented that inflammation, apoptosis and necrosis, hypoxic injury and production of reactive oxygen species are involved in the pathogenesis of IRI (8). The modulation of inflammatory response, inhibition of apoptosis and amelioration of oxidative stress confer an advantage to the prevention and treatment of IRI $(9,10)$. Erythropoietin (EPO) has been found to confer such an advantage $(11,12)$, but the underlying molecular mechanism remains unclear.

Inflammation is invariably found to be an important initiating and aggravating factor in both acute and chronic kidney injury (13). Nuclear factor- $\kappa \mathrm{B}(\mathrm{NF}-\kappa \mathrm{B})$, a pivotal mediator of the inflammatory response, modulates the expression levels of adhesion molecules, chemokines and other pro-inflammatory molecules in the kidney $(14,15)$. It was reported that interleukin (IL)-4 promoted the activation of signal transducer and activator of transcription 6 (STAT6), suppressing the transcriptional activation of NF- $\mathrm{KB}$-dependent proinflammatory mediators following liver IRI $(16,17)$. Apoptosis is a principal cause of cell death in the kidney following IRI $(18,19)$. Apoptosis-related proteins, such as caspase-3, play important roles in renal IRI $(20,21)$. Therefore, regulating inflammation and cell death is a promising therapeutic strategy for reversing IRI and protecting renal allografts.

EPO is a hematopoietic hormone produced by the kidney and fetal liver in response to hypoxia, inflammation and cell death (22). EPO exerts numerous protective effects. Importantly, 
EPO may exert antioxidant, anti-inflammatory and anti-apoptotic effects against IRI in the brain (23) and kidney (24). It was demonstrated that, under conditions of renal IRI, the expression levels of EPO in the kidney were decreased (25). A variety of signal transduction pathways, including mitogen-activated protein kinase (MAPK) and $\mathrm{NF}-\kappa \mathrm{B}$, were involved in the EPO-mediated cytoprotective effects $(26,27)$. The cell-protective effect of EPO was significantly attenuated by pretreatment with the specific p-p38 inhibitor, suggesting that MAPK pathways may be responsible for cell survival under cytotoxic conditions (26). EPO treatment significantly decreased the lipopolysaccharide-induced elevation of creatinine $(\mathrm{Cr})$ and $\mathrm{NF}-\kappa \mathrm{B}$, indicating that EPO may play a protective role against IRI by reducing the inflammatory response and tissue degeneration, possibly via the NF- $\kappa \mathrm{B}$ signaling pathway (27). Therefore, the mechanisms underlying the protective effect of EPO against renal IRI remain to be fully elucidated.

The aim of the present study was to investigate the effect of EPO on the levels of chemokines, including interferon (IFN) $-\gamma$, IL-4, IL-10 and Cr, by the siRNA technique, and determine whether the renoprotective effect of EPO against IRI is exerted through the STAT6/MAPK/NF- $\mathrm{B}$ pathway by using specific inhibitors, including the STAT6 inhibitor AS1517499, the JNK inhibitor SP600125, the p38 MAPK inhibitor SB203580, and the NF- $\kappa \mathrm{B}$ inhibitor lactacystin. This detailed protective mechanism of EPO may improve reperfusion tolerance in ischemic kidneys and benefit transplant recipients.

\section{Materials and methods}

Animals. Specific pathogen-free (SPF) adult male Lewis rats $(\mathrm{n}=64)$ and Brown Norway rats $(\mathrm{n}=64), 12-16$ weeks old, weighing 250-350 g were purchased from Charles River Laboratories (Beijing, China). All animal experiments were performed in accordance with the Experimental Animal Regulations established by The Ministry of Science and Technology of the People's Republic of China, and the Guidelines for the Care and Use of Laboratory Animals published by the National Institutes of Health (Bethesda, MD, USA). The study received ethical approval from the Ethics Committee of Sun Yat-sen University. Prior to performing the experiments, all the animals were subjected to an overnight fast with unlimited access to water.

Establishment of the animal model. The SPF rats were randomly allocated to three groups (control, EPO and EPO-siRNA; $n=8$ ). The Lewis rats were used as the recipients, and the Brown Norway rats were used as donors in the renal transplants. Kidney transplantation was performed as previously described (28). In brief, the rats were anesthetized with inhalation of $10 \%$ chloral hydrate $0.3 \mathrm{ml} / 100 \mathrm{~g}$ body weight. The left kidney was prepared by freeing the ureter from the attachments. The donor kidney and ureter were removed en bloc. Donor kidney grafts were washed with $4^{\circ} \mathrm{C}$ saline solution and stored in $4^{\circ} \mathrm{C}$ HCA solution. The right kidney was removed from the recipient, and the donor kidney was transplanted. An anastomosis was created between the donor and recipient renal artery, renal vein and ureters with end-to-end anastomosis, to establish the unilateral orthotopic renal transplantation rat model. Rat rectal temperature was maintained at $35-37^{\circ} \mathrm{C}$ during surgery. After surgery, the rats were placed on a heat pad until they recovered.
Table I. Primer sequences used for RT-qPCR.

\begin{tabular}{ll}
\hline Genes & \multicolumn{1}{c}{ Primer sequences (5'-3') } \\
\hline GAPDH & F: GGTGAAGGTCGGTGTGAAC \\
GAPDH & R: CCTTGACTGTGCCGTTGAA \\
EPO & F: AGAATGAAGGTGGAAGAACAGG \\
EPO & R: CCCGAAGCAGTGAAGTGAGG \\
IFN- $\gamma$ & F: GAACTGGCAAAAGGACGGTAA \\
IFN- $\gamma$ & R: AACTTGGCGATGCTCATGAAT \\
IL-4 & F: CCACCTTGCTGTCACCCTGT \\
IL-4 & R: CCGTGGTGTTCCTTGTTGC \\
IL-10 & F: CTATGTTGCCTGCTCTTACTGG \\
IL-10 & R: ATGTGGGTCTGGCTGACTGG
\end{tabular}

RT-qPCR, reverse transcription-polymerase chain reaction; F, forward; $\mathrm{R}$, reverse; EPO, erythropoietin; IFN, interferon; IL, interleukin.

Experimental design. After establishment of the unilateral orthotopic renal transplantation rat model, continuous administration of saline solution, recombinant human EPO (500 U/kg), or lentivirus-mediated EPO siRNA ( $1 \times 10^{7}$ infectious units) (sc-37220-V; Santa Cruz Biotechnology, Inc., Santa Cruz, CA, USA) was performed, once a day for 7 days. After the last administration, the rats were sacrificed and the kidneys were removed and processed for immunohistochemistry (IHC) and immunofluorescence (IFC) assessment of EPO levels, hematoxylin and eosin (H\&E), periodic acid-Schiff (PAS), PAS-methenamine (PASM) and Masson's trichrome staining, for detection of the levels of creatinine (Cr), EPO, IL-4, IL-10 and IFN- $\gamma$, and for western blot analysis of thymic stromal lymphopoietin (TSLP), STAT6, NF- $\mathrm{B}, \mathrm{JNK}$, p38, and caspase-1 and -3 in renal tissues.

To further investigate the role of the STAT6/MAPK/NF- $\kappa$ B pathway in EPO-mediated renoprotection. The other 40 rats were divided into 5 groups (control, and 4 groups of the different inhibitors; $n=8)$. The animals received the STAT6 inhibitor AS1517499 (10 mg/kg), the JNK inhibitor SP600125 (30 mg/kg), the p38 MAPK inhibitor SB203580 (15 mg/kg), or the NF- $\mathrm{NB}$ inhibitor lactacystin $(10 \mathrm{mg} / \mathrm{kg})$ in the presence of EPO and using the same route of administration of EPO following renal transplantation. The rats were then sacrificed and the kidneys were removed and processed for H\&E, PAS, PASM, and Masson's trichrome staining, for detection of the levels of Cr, EPO, IL-4, IL-10 and IFN- $\gamma$, and for western blot analysis of TSLP, STAT6, NF- $\kappa$ B, JNK, p38, and caspase-1 and -3 in renal tissues.

IHC and IFC. The kidneys were processed for IHC and IFC according to standard protocols. For IHC, $3-\mu \mathrm{m}$ paraffin sections using rabbit anti-mouse EPO monoclonal antibody (1:100; sc-5290) and goat anti-rabbit IgG poly-HRP (1:100; sc-2004) (both from Santa Cruz Biotechnology, Inc.). For IFC, the sections were fixed in $4 \%$ paraformaldehyde and incubated with rabbit anti-human EPO monoclonal antibody (1:50; sc-80995) and FITC-labeled goat anti-rabbit secondary antibody (1:150; sc-2004) (both from Santa Cruz Biotechnology, Inc.). The fluorescence intensity of EPO was calculated by using ImageJ software (National Institutes of Health). For 

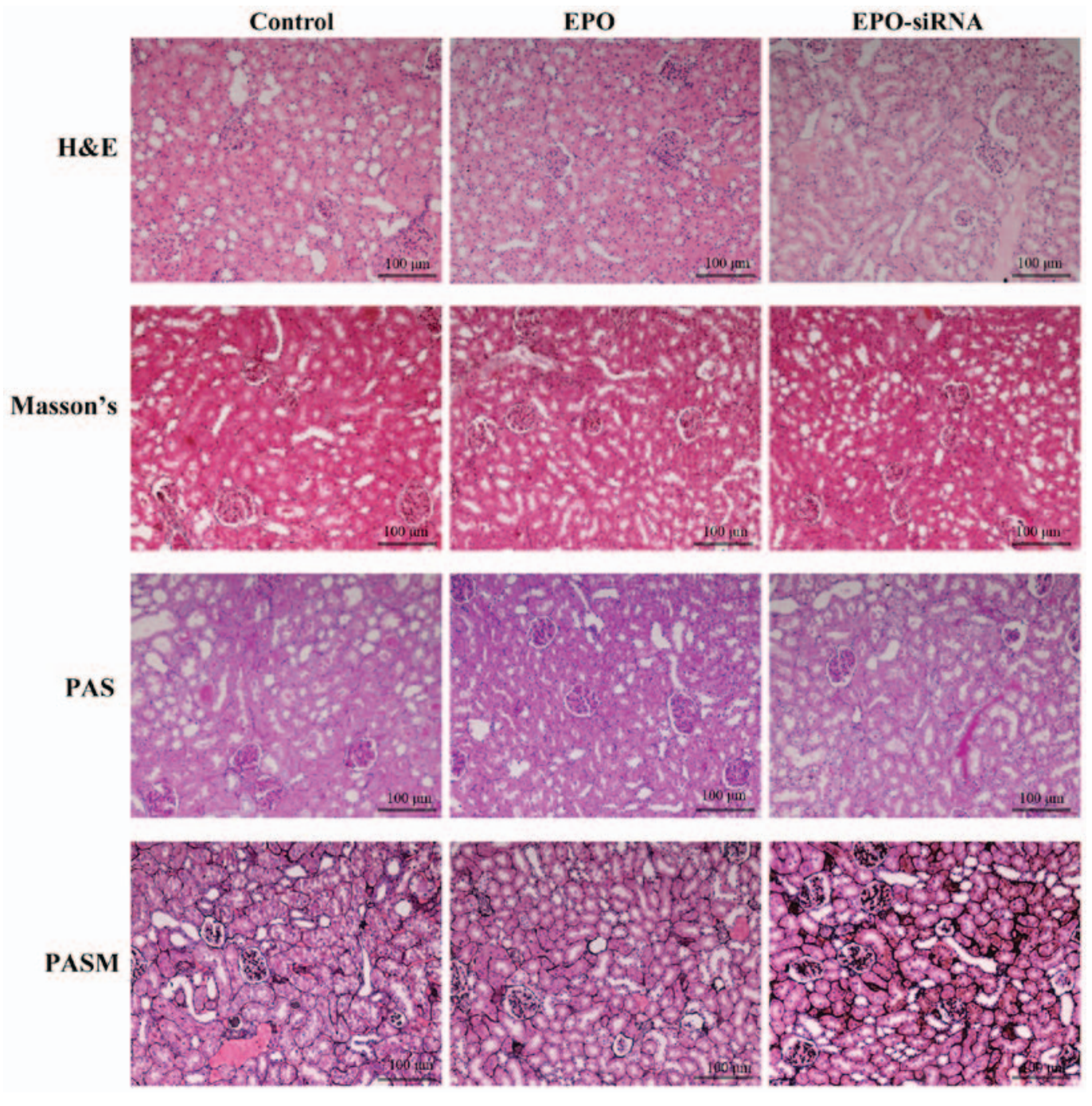

Figure 1. Histological analysis revealed pathological changes in the kidneys. The unilateral orthotopic renal transplantation rat model included administration of saline solution (control), recombinant human erythropoietin (EPO; $500 \mathrm{U} / \mathrm{kg}$ ), or lentivirus-mediated EPO-siRNA. The kidneys were processed for hematoxylin and eosin (H\&E), periodic acid-Schiff (PAS), PAS-methenamine (PASM) and Masson's trichrome staining.

nuclear staining, DAPI was used and confocal images were acquired as previously described $(29,30)$ using the LSM 510 microscope (Carl Zeiss AG, Oberkochen, Germany). Formalin-fixed paraffin-embedded tissues were also stained with H\&E and PAS, PASM and Masson's trichrome staining.

Reverse transcription-quantitative polymerase chain reaction $(R T-q P C R)$. Total RNA was extracted with TRIzol reagent (Invitrogen; Thermo Fisher Scientific, Carlsbad, CA, USA) from homogenated whole kidneys. RNA was reverse transcribed to cDNA using SuperScript II Reverse Transcriptase (Invitrogen; Thermo Fisher Scientific). RT-qPCR was performed on an ABI Prism 5700 Sequence Detection system (Applied Biosystems, Foster City, CA, USA) with SYBR-Green PCR Core reagents. The primers are listed in Table I. GAPDH served as internal control. The relative mRNA expression levels of each target gene were normalized to those of the controls using the $2^{-\Delta \Delta \mathrm{Cq}}$ method.

Enzyme-linked immunosorbent assay (ELISA). Serum Cr, EPO, IL-4, IL-10 and IFN- $\gamma$ levels were detected in a 96-well microtiter plate by using a commercial ELISA kit (Biosource, Camarillo, CA, USA) according to the manufacturer's instruc- tions. All samples were tested in duplicate. The plate was read at $450 \mathrm{~nm}$ on a microplate reader (BioTek Instruments, Inc., Winooski, VT, USA). The levels were calculated from a standard curve and expressed in $\mathrm{pg} / \mathrm{ml}$.

Western blot analysis. The protein lysates were prepared with RIPA and subjected to three freeze/thaw cycles using liquid nitrogen. The lysates were then electrophoresed in $10 \%$ SDS-PAGE (Bio-Rad Laboratories, Inc., Hercules, CA, USA), transferred to nitrocellulose membrane, blocked with $0.5 \%$ skimmed milk and incubated with TSLP, STAT6, NF-kB, JNK, p38 and caspase- 1 and -3 primary antibodies (all from Santa Cruz Biotechnology, Inc.) for $1 \mathrm{~h}$. Polyclonal anti-GAPDH was used as an internal control. After three washes, the membrane was incubated with horseradish peroxidase-conjugated IgG and developed under chemiluminescence condition (Amersham Pharmacia Biotech, Piscataway, NJ, USA) using ChemiDoc XRS (Bio-Rad Laboratories, Inc.). The quantification and analysis for the blots were performed with ImageJ software.

Statistical analysis. All data are presented as means \pm standard deviation. Data between groups were compared using 
A

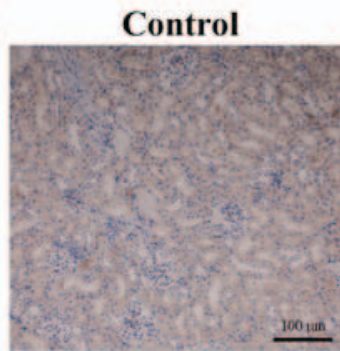

EPO

EPO-siRNA
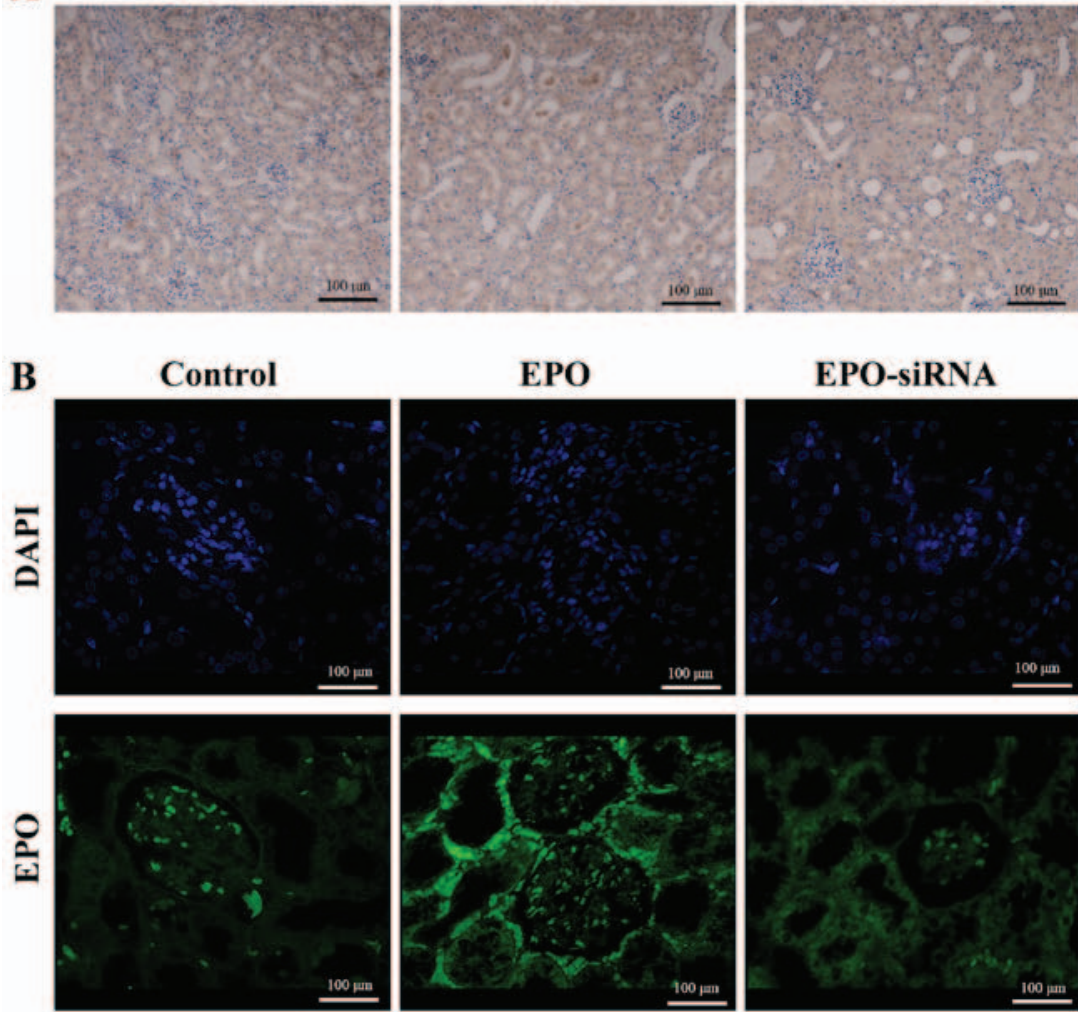

EPO-siRNA
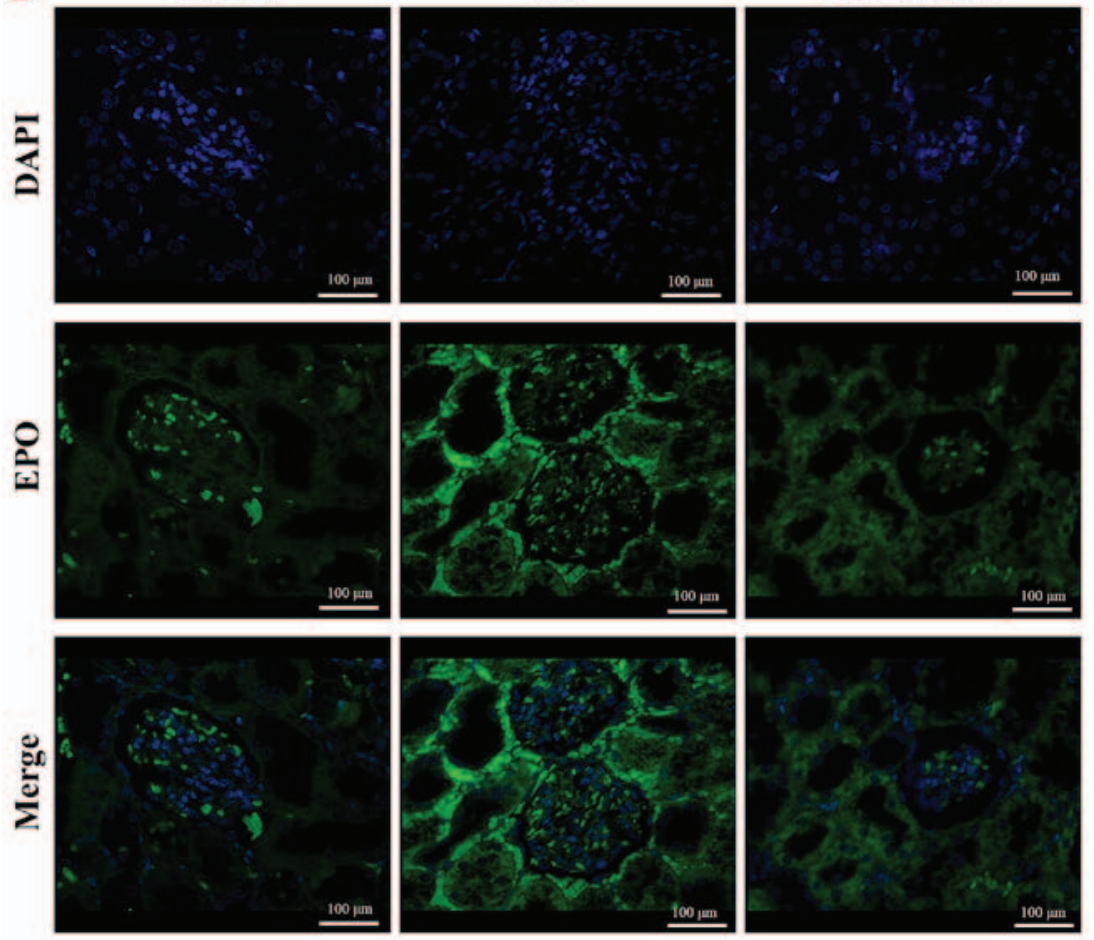

C

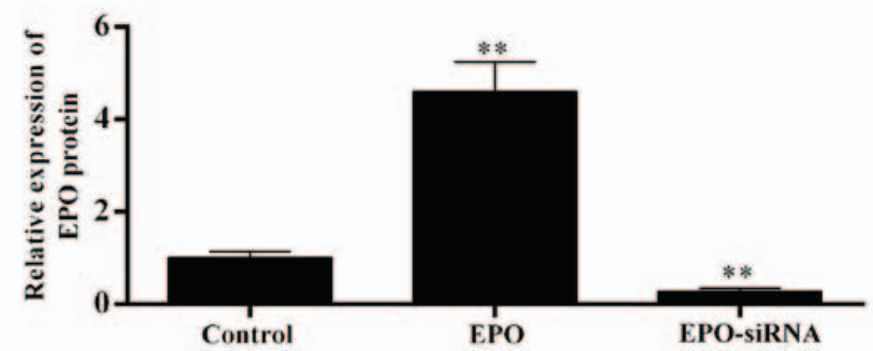

Figure 2. Level of erythropoietin (EPO) in the kidneys. (A) Immunohistochemistry, (B) immunofluorescence and (C) qualification. The unilateral orthotopic renal transplantation rat model was etsbalished with administration of saline solution (control), recombinant human EPO (500 U/kg), or lentivirus-mediated EPO-siRNA. ${ }^{* *} \mathrm{P}<0.01$ vs. control.

two-tailed unpaired t-test or analysis of variance. $\mathrm{P}<0.05$ was considered to indicate statistically significant differences. All analyses were performed using SPSS 16.0 software (SPSS Inc., Chicago, IL, USA).

\section{Results}

Pathological changes of the kidneys. Following continuous administration of saline solution, recombinant human EPO (500 U/kg), or lentivirus-mediated EPO-siRNA for 7 days, histological analysis of kidneys was performed (Fig. 1). The images of H\&E, PAS, PASM and Masson's staining revealed that IRI occurred after renal transplantation. Infiltration of inflammatory cells, with a large number of lymphocytes around the blood vesselst was observed. Furthermore, EPO protected the kidney from IRI, through decreasing the extent of tissue congestion and inflammatory cell infiltration; however, EPO-siRNA did not exert the same protective effect.

The level of EPO was detected in the kidneys (Fig. 2). Both IHC (Fig. 2A) and IFC images (Fig. 2B), and data qualification (Fig. 2C) revealed that the EPO levels in EPO-treated rats were higher compared with those in the saline solution and EPO-siRNA groups. Thus, the EPO level appears to be inversely correlated to the renal IRI. 

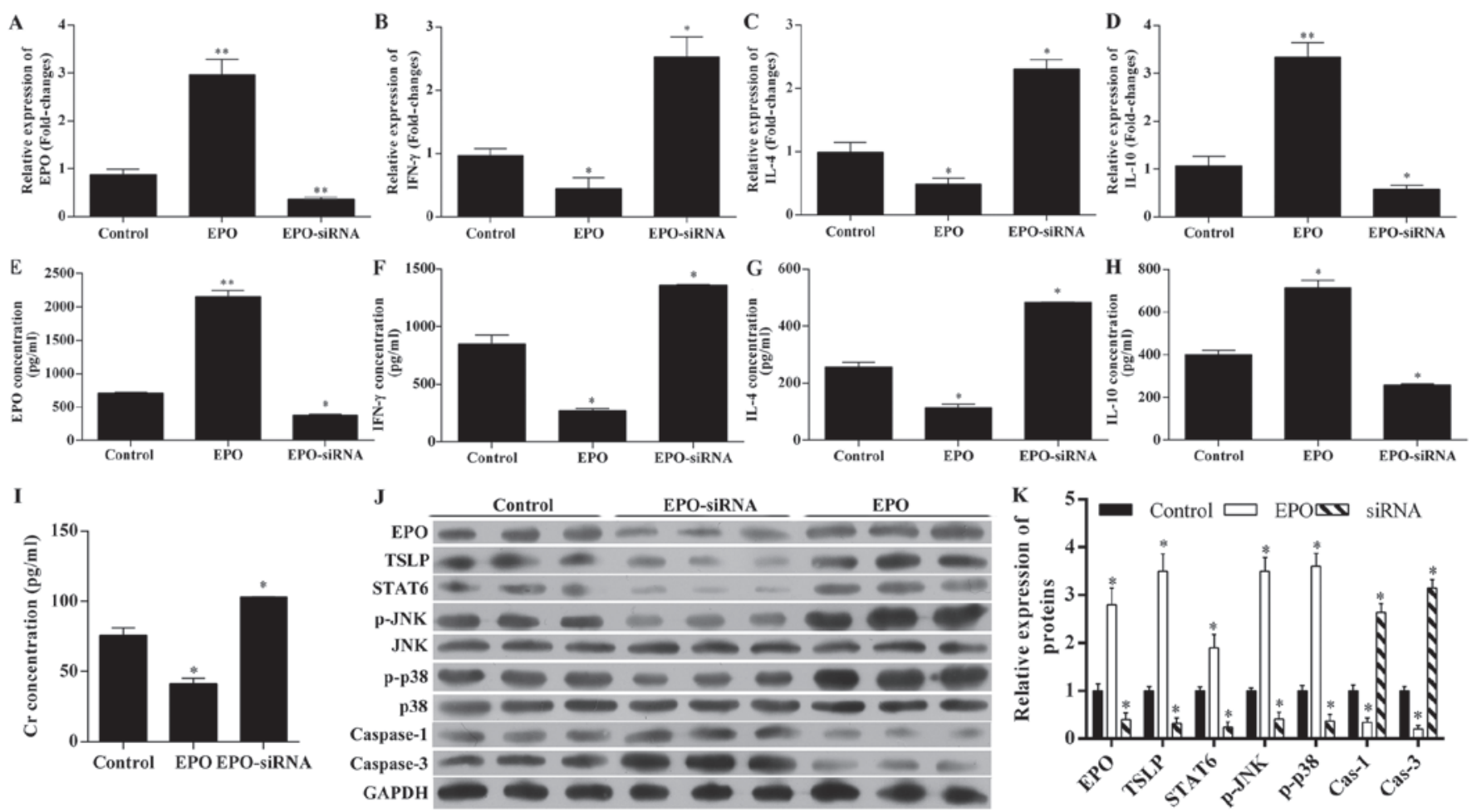

Figure 3. Changes in the levels of erythropoietin (EPO), interferon (IFN)- $\gamma$, interleukin (IL)-4, IL-10, creatinine (Cr), thymic stromal lymphopoietin (TSLP), signal transducer and activator of transcription 6 (STAT6), p-JNK, p-p38, and caspase-1 and -3. The unilateral orthotopic renal transplantation rat model was established by administration of saline solution (control), recombinant human EPO (500 U/kg), or lentivirus-mediated EPO-siRNA. Using RT-qPCR, the expression of (A) EPO, (B) IFN- $\gamma$, (C) IL-4 and (D) IL-10 in renal tissues were detected. Using ELISA, the serum level of (E) EPO, (F) IFN- $\gamma$, (G) IL-4, (H) IL-10 and (I) Cr were detected. (J) Using western blot analysis, the expression of EPO, TSLP, STAT6, p-JNK, p-p38 and caspase-1 and -3 were also detected and $(\mathrm{K})$ quantified. ${ }^{*} \mathrm{P}<0.05 ;{ }^{* *} \mathrm{P}<0.01$ vs. control. RT-qPCR, reverse transcription-quantitative polymerase chain reaction.

EPO downregulates the expression of IFN- $\gamma, I L-4$ and $\mathrm{Cr}$ and upregulates the expression of IL-10, and this effect is reversed by EPO-siRNA. The expression of EPO, IFN- $\gamma$, IL-4 and IL-10 in tissues and serum were detected by RT-qPCR and ELISA, respectively (Fig. 3A-G). As expected, EPO tissue and serum levels were significantly upregulated in rats with EPO administration, and was significantly downregulated by EPO-siRNA (Fig. 3A and E). EPO treatment reduced the expression of IFN- $\gamma$ (Fig. 3B and F) and IL-4 (Fig. 3C and G), as well as the serum levels, whereas it increased the expression of IL-10 at the mRNA level and in the serum (Fig. 3D and H). All these effects induced by EPO were reversed by EPO-siRNA.

The $\mathrm{Cr}$ level in the serum was also investigated (Fig. 3I). The results revealed that, compared with controls, EPO administration decreased the serum level of $\mathrm{Cr}$, and EPO-siRNA increased the serum level of $\mathrm{Cr}$.

EPO upregulated the expression of TSLP, STAT6, $p$-JNK and p-p38, and downregulated the expression of caspase-3, while EPO-siRNA exerted the opposite effects. To analyze the underlying molecular mechanisms for EPO-mediated renoprotection, the changes in TSLP and STAT6/MAPK/NF- $\mathrm{B}$ pathway were detected by western blot analysis (Fig. 3J and K). EPO administration upregulated the expression of TSLP, STAT6, p-JNK, p-p38, but downregulated the expression of caspase-3. By contrast, EPO-siRNA decreased the expression of EPO, TSLP, STAT6, p-JNK, p-p38 and caspase-1, but increased the expression of caspase-3, indicating that EPO protects the kidney from IRI via the STAT6/MAPK/NF- $\kappa$ B signaling pathway.

Assessment of the role of the STAT6/MAPK/NF- $\kappa B$ pathway in EPO-mediated renoprotection from IRI. We further investigated whether the STAT6 inhibitor AS1517499, the JNK inhibitor SP600125, the p38 MAPK inhibitor SB203580, or the NF- $\mathrm{B}$ inhibitor lactacystin suppressed the effects of EPO in inhibiting renal IRI (Figs. 4 and 5). The histological analysis revealed that EPO-mediated renoprotection was obviously inhibited by AS1517499, SP600125, SB203580 and lactacystin (Fig. 4).

The chemokine levels were also measured (Fig. 5A-G). IFN- $\gamma$ (Fig. 5A and C) and IL-4 (Fig. 5B and D) at the mRNA level and in the serum levels were significantly increased by these inhibitors in the presence of EPO, while IL-10 levels (Fig. 5C and F) were significantly decreased in the presence of EPO. The serum level of $\mathrm{Cr}$ in the presence of EPO was also increased by each of these inhibitors (Fig. 5G).

The STAT6 inhibitor AS1517499 inhibited the expression of STAT6, p-JNK, p-p38 and p65, while it increased the expression of caspase- 1 and -3 in the presence of EPO (Fig. $5 \mathrm{H}$ and I), suggesting that STAT6 acted upstream of MAPK (p-JNK and p-p38) and NF- $\mathrm{B}$. The JNK inhibitor SP600125 and the p38 MAPK inhibitor SB203580 inhibited the expression of $\mathrm{p}-\mathrm{JNK}$ and $\mathrm{p} 65$, while they increased the expression of caspase- 1 and -3 in the presence of EPO (Fig. 5J and K), suggesting that MAPK (p-JNK and p-p38) acted upstream 


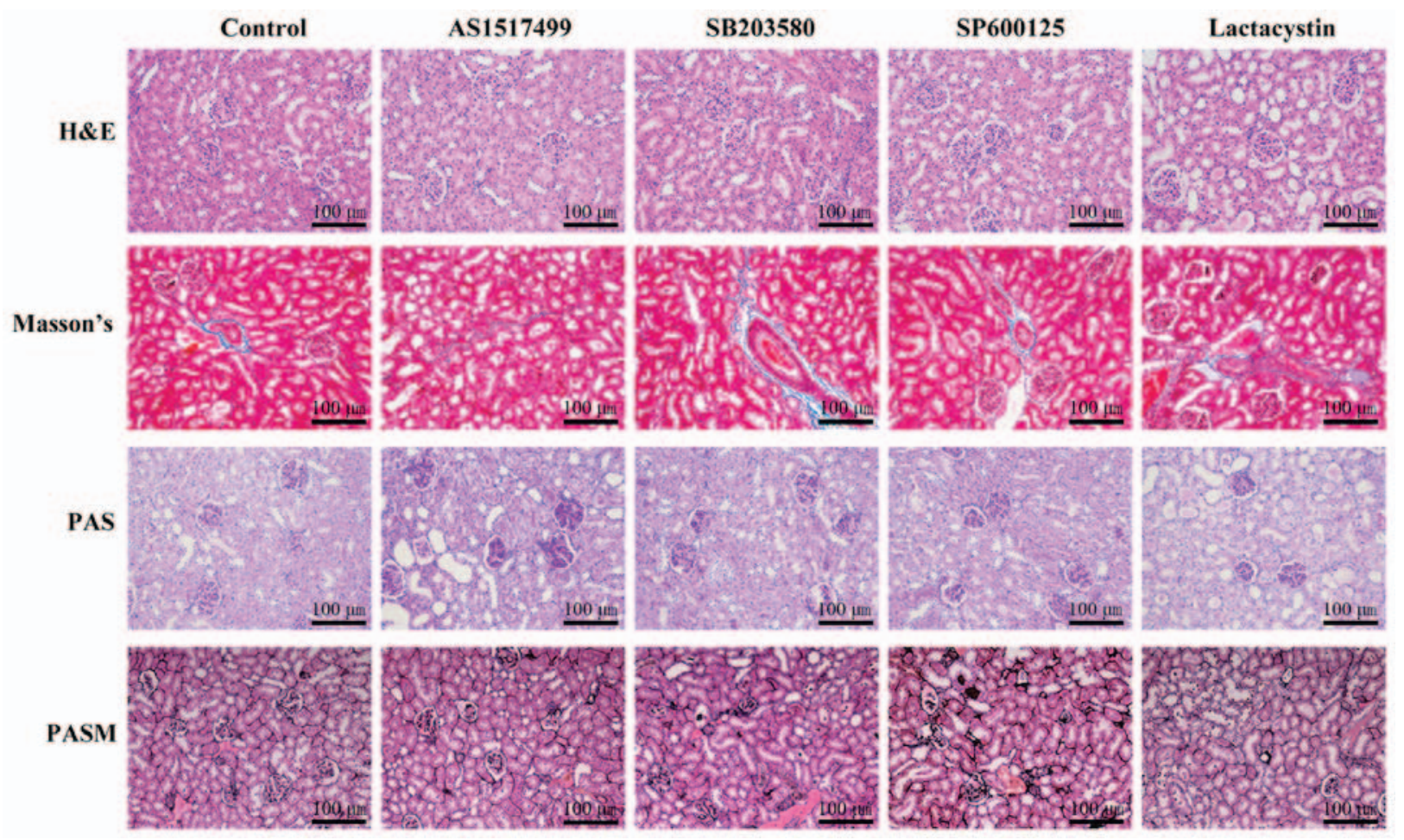

Figure 4. Histological analysis of the signal transducer and activator of transcription 6 (STAT6)/mitogen-activated protein kinase (MAPK)/nuclear factor- $\mathrm{\kappa B}(\mathrm{NF}-\mathrm{\kappa B})$ pathway in erythropoietin (EPO)-mediated renoprotection from ischemia/reperfusion injury. Rats with unilateral orthotopic renal transplantation received the STAT6 inhibitor AS1517499, the JNK inhibitor SP600125, the p38 MAPK inhibitor SB203580, or the NF- $\mathrm{BB}$ inhibitor lactacystin in the presence of EPO. Rats administered EPO served as control.

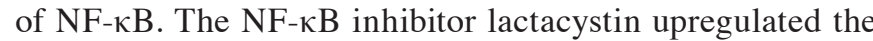
expression of caspase-1 and -3 in the presence of EPO (Fig. 5L).

Therefore, all the inhibitors of STAT6, JNK, p38 MAPK and $\mathrm{NF}-\mathrm{\kappa B}$ reversed the changes in the chemokine levels, $\mathrm{Cr}$ levels, and caspase-1 and -3 expression. These results suggest that the STAT6/MAPK/NF-кB signaling pathway was involved in EPO-mediated renoprotection from IRI.

\section{Discussion}

Recent studies suggested that the MAPK/NF- $\mathrm{KB}$ signaling pathway is involved in the EPO-mediated protection from IRI following renal transplantation $(26,27)$, and that the STAT6/NF-кB signaling pathway is involved in the inflammatory responses $(16,17)$. Moreover, apoptosis is known to play a key role in renal IRI $(18,19)$. Apoptosis-related proteins, such as caspase-3, may be involved in renal IRI $(20,21)$. However, the role of the STAT6/MAPK/NF- $\mathrm{BB}$ signaling pathway in EPO-induced renoprotection remains poorly understood. In the present study, a sham-operation group was not constructed; all experiments were performed following establishment of the unilateral orthotopic renal transplantation rat model. We demonstrated that recombinant human EPO protected against IRI following renal transplantation by reducing the inflammatory responses and promoting cell survival under cytotoxic conditions. Specific inhibitors, including the STAT6 inhibitor AS1517499, the JNK inhibitor SP600125, the p38 MAPK inhibitor SB203580, and the NF-kB inhibitor lactacystin, reversed the effects of EPO, further suggesting the importance of the STAT6/MAPK/NF- $\mathrm{KB}$ signaling pathway in EPO-mediated renoprotection from IRI.
EPO is produced by the kidney and fetal liver and has long been considered as merely a factor modulating erythropoiesis. However, recent studies demonstrated that EPO may also exert protective effects against hypoxia, inflammation and cell death following IRI in the kidney (27), brain (31), and myocardium (32-34). Our results demonstrated that EPO protected the kidney from IRI, through decreasing the extent of tissue congestion and inflammatory cell infiltration; however, EPO-siRNA did not exert such a protective effect. IHC (Fig. 2A) and IFC (Fig. 2B) images revealed that the EPO levels in EPO-treated rats were higher compared with those the the saline solution and EPO-siRNA groups, suggesting that the EPO level is inversely correlated with renal IRI.

EPO increased the levels of anti-inflammatory cytokines, such as IL-10, while it reduced the levels of pro-inflammatory cytokines, such as IFN- $\gamma$ and IL-4, and Cr. This is consistent with previous studies demonstrating that treatment with anti-inflammatory cytokine IL-10 reduced renal and systemic inflammation (35), and reduced the serum levels of proinflammatory cytokine IFN- $\gamma$, protecting the kidney from injury (36). These data suggest that EPO not only suppressed the expression of pro-inflammatory cytokines, but also increased the level of anti-inflammatory cytokines. TLSP is a critical cytokine that exacerbates allergic and fibrotic reactions (37). TLSP is mainly produced by epithelial cells and its production is induced by inflammatory cytokines (38). NF- $\kappa \mathrm{B}$ also regulates TSLP mRNA expression (38). TSLP was highly expressed in the kidney in the presence of EPO, and this was inhibited by EPO-siRNA, indicating that TSLP may be involved in EPO-mediated protection from IRI. 

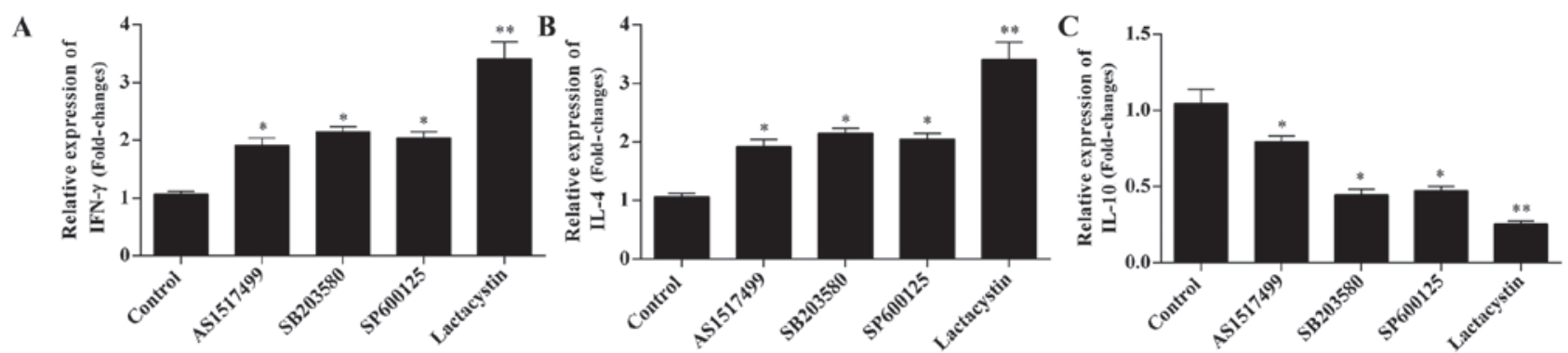

D
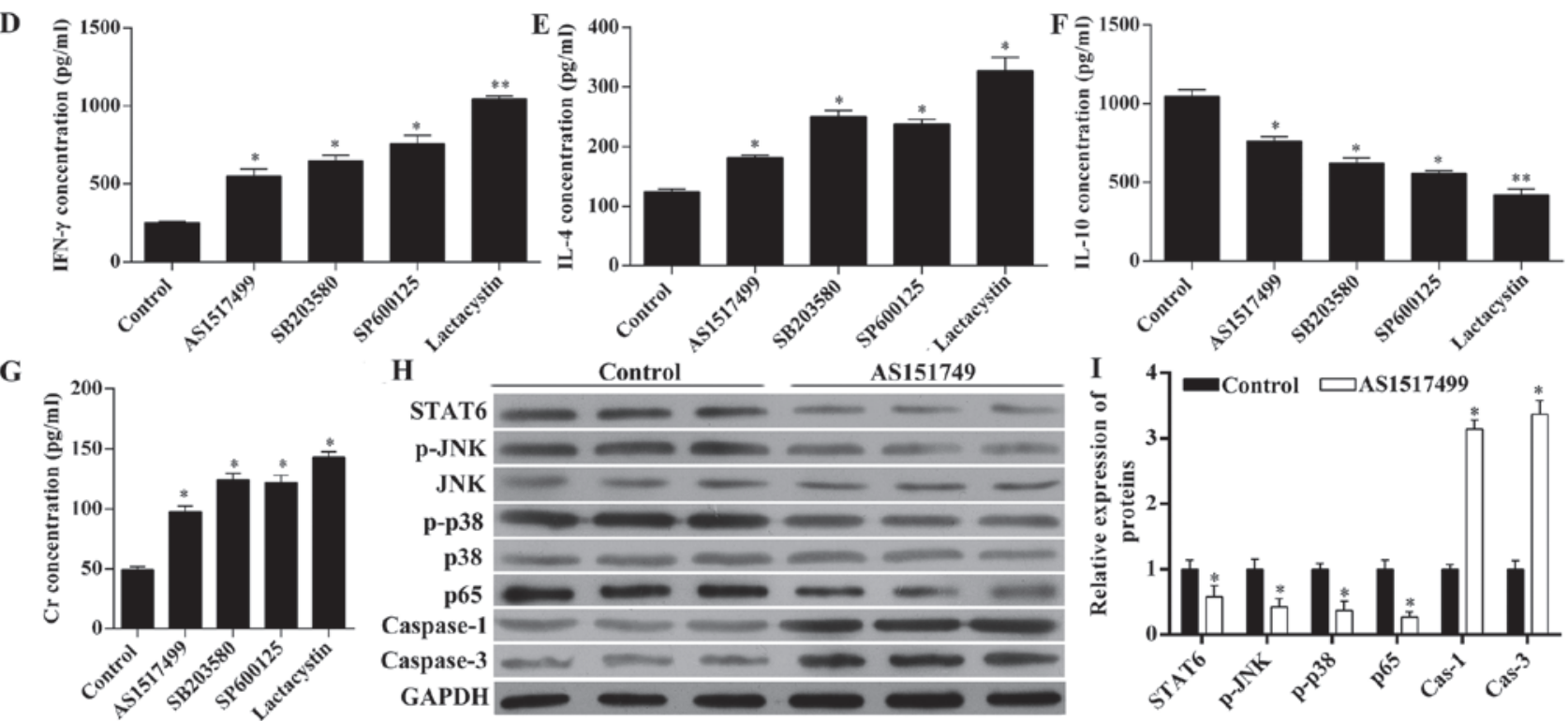

$\mathbf{J}$

Control

SP600125

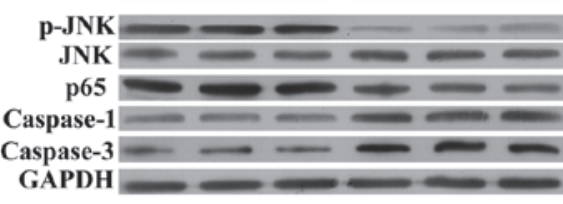

$\mathbf{K}$

Control
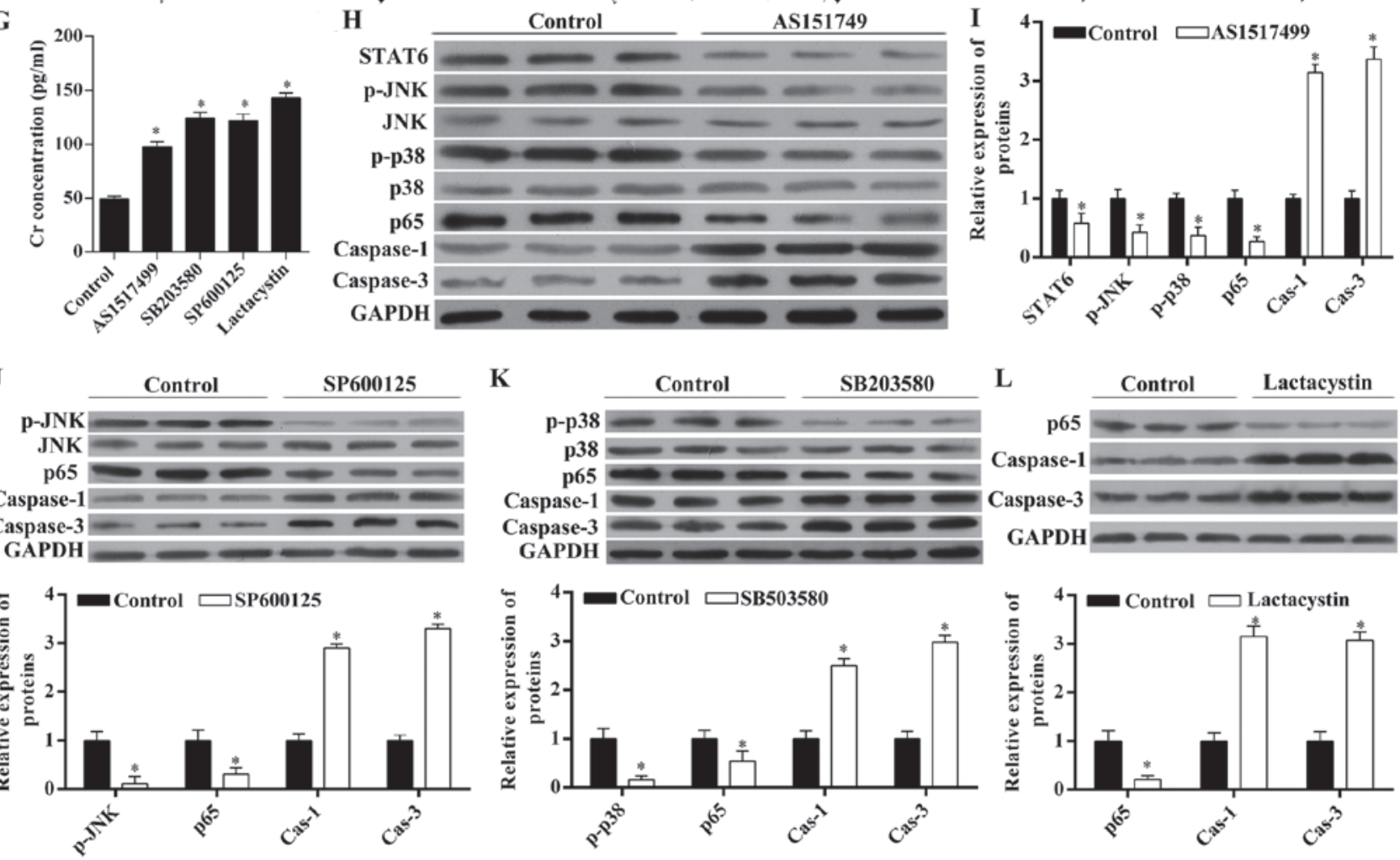

Figure 5. Erythropoietin (EPO) modulates the levels of interferon (IFN)- $\gamma$, interleukin (IL)-4, IL-10, creatinine (Cr) and caspase-1 and -3 via the signal transducer

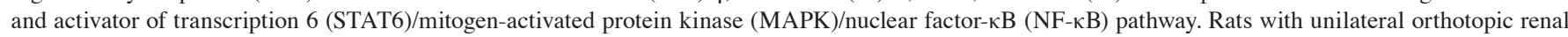
transplantation received the STAT6 inhibitor AS1517499, the JNK inhibitor SP600125, the p38 MAPK inhibitor SB203580, or the NF- $\mathrm{BB}$ inhibitor lactacystin, in the presence of EPO. Rats administered EPO were used as control. Using RT-qPCR, the expression of (A) IFN- $\gamma$, (B) IL-4 and (C) IL-10 in renal tissues was detected. Using ELISA, the serum level of (D) IFN- $\gamma$, (E) IL-4, (F) IL-10 and (G) Cr was detected. (H) Using western blot analysis, the expression of STAT6, p-JNK, p-p38, p65 and caspase-1 and -3 with concurrent administration of EPO and AS1517499 were detected and (I) quantified. The (J) expression of p-JNK, p65 and caspase-1 and -3 with administration of EPO and SP600125; (K) expression of p-p38, p65 and caspase-1 and -3 with administration of EPO and SB203580; and the (L) expression of p65 and caspase- 1 and -3 with administration of EPO and lactacystin were detected and quantified. ${ }^{*} \mathrm{P}<0.05$ and ${ }^{* *} \mathrm{P}<0.01$ vs. control.

It has been reported that MAPK and NF- $\mathrm{kB}$ played an important role in EPO-mediated cytoprotective effects $(26,27)$. EPO significantly decreased the lipopolysaccharide-induced elevation of $\mathrm{Cr}$ and $\mathrm{NF}-\kappa \mathrm{B}$ levels, indicating that EPO may play a protective role against IRI by reducing the inflammatory response and tissue degeneration, possibly via the NF- $\mathrm{\kappa B}$ signaling pathway (27). Furthermore, STAT6 was elevated by inflammatory cytokines, such as IL-4, and then suppressed the transcriptional activation of NF- $\kappa \mathrm{B}-$ dependent proinflammatory mediators following liver IRI $(16,17)$. In the present study, the specific inhibitors of STAT6, JNK, p38 MAPK and NF- $\mathrm{KB}$ reversed the EPO-induced changes in the levels of IFN- $\gamma$, IL-4, IL-10, $\mathrm{Cr}$ and TSLP. In addition, MAPK (p-JNK and p-p38) acted upstream of NF- $\mathrm{NB}$, and NF- $\mathrm{NB}$ signaling regulated the expression of caspase- 1 and -3 , which may responsible for the cytotoxicity observed under IRI conditions. 
In conclusion, the present study demonstrated that EPO exerted a protective effect in renal IRI via the STAT6/MAPK/NF-кB pathway.

\section{Acknowledgements}

The present study was supported by the National Natural Science Foundation of China (grant nos. 81470977 and 81270835), the Science and Technology Planning Project of Guangdong Province, China (grant no. 2014A020212121), and The Basic Service Charge Young Teachers Cultivation Project of Sun Yat-sen University (grant no. 13ykpy35).

\section{References}

1. Naylor KL, Zou G, Leslie WD, Hodsman AB, Lam NN McArthur E, Fraser LA, Knoll GA, Adachi JD, Kim SJ, et al: Risk factors for fracture in adult kidney transplant recipients. World J Transplant 6: 370-379, 2016.

2. Wolfe RA, Ashby VB, Milford EL, Ojo AO, Ettenger RE, Agodoa LY, Held PJ and Port FK: Comparison of mortality in al patients on dialysis, patients on dialysis awaiting transplantation, and recipients of a first cadaveric transplant. N Engl J Med 341: $1725-1730,1999$

3. Kosieradzki M and Rowiński W: Ischemia/reperfusion injury in kidney transplantation: mechanisms and prevention. Transplant Proc 40: 3279-3288, 2008.

4. Hoshinaga K, Shiroki R, Fujita T, Kanno T and Naide Y: The fate of 359 renal allografts harvested from non-heart beating cadaver donors at a single center. Clin Transpl: 213-220, 1998.

5. Perico N, Cattaneo D, Sayegh MH and Remuzzi G: Delayed graft function in kidney transplantation. Lancet 364: 1814-1827, 2004

6. Gueler F, Gwinner W, Schwarz A and Haller H: Long-term effects of acute ischemia and reperfusion injury. Kidney Int 66: 523-527, 2004

7. Bonventre JV and Yang L: Cellular pathophysiology of ischemic acute kidney injury. J Clin Invest 121: 4210-4221, 2011.

8. Devarajan P: Update on mechanisms of ischemic acute kidney injury. J Am Soc Nephrol 17: 1503-1520, 2006.

9. Bajwa A, Kinsey GR and Okusa MD: Immune mechanisms and novel pharmacological therapies of acute kidney injury. Curr Drug Targets 10: 1196-1204, 2009.

10. Silver SA, Cardinal H, Colwell K, Burger D and Dickhout JG: Acute kidney injury: preclinical innovations, challenges, and opportunities for translation. Can J Kidney Health Dis 2: 30, 2015

11. Liu QS, Cheng ZW, Xiong JG, Cheng S, He XF and Li XC: Erythropoietin pretreatment exerts anti-inflammatory effects in hepatic ischemia/reperfusion-injured rats via suppression of the TLR2/NF-кB pathway. Transplant Proc 47: 283-289, 2015.

12. McCook O, Georgieff M, Scheuerle A, Möller P, Thiemermann C and Radermacher P: Erythropoietin in the critically ill: do we ask the right questions? Crit Care 16: 319, 2012.

13. Akcay A, Nguyen Q and Edelstein CL: Mediators of inflammation in acute kidney injury. Mediators Inflamm 2009: 137072, 2009.

14. Kono H, Nakagawa K, Morita S, Shinoda K, Mizuno R, Kikuchi E, Miyajima A, Umezawa K and Oya M: Effect of a novel nuclear factor- $\kappa \mathrm{B}$ activation inhibitor on renal ischemia-reperfusion injury. Transplantation 96: 863-870, 2013.

15. Latanich CA and Toledo-Pereyra LH: Searching for NF-kappaB-based treatments of ischemia reperfusion injury. J Invest Surg 22: 301-315, 2009.

16. Bennett BL, Cruz R, Lacson RG and Manning AM: Interleukin-4 suppression of tumor necrosis factor alpha-stimulated E-selectin gene transcription is mediated by STAT6 antagonism of NF-kappaB. J Biol Chem 272: 10212-10219, 1997.

17. Kato A, Yoshidome H, Edwards MJ and Lentsch AB: Reduced hepatic ischemia/reperfusion injury by IL-4: potential anti-inflammatory role of STAT6. Inflamm Res 49: 275-279, 2000.

18. Yang Z, Zhong Z, Li M, Xiong Y, Wang Y, Peng G and Ye Q: Hypothermic machine perfusion increases A20 expression which protects renal cells against ischemia/reperfusion injury by suppressing inflammation, apoptosis and necroptosis. Int J Mol Med 38: 161-171, 2016.

19. Toronyi E: Role of apoptosis in the kidney after reperfusion. Orv Hetil 149: 305-315, 2008 (In Hungarian).
20. Hu H, Jiang W, Xi X, Zou C and Ye Z: MicroRNA-21 attenuates renal ischemia reperfusion injury via targeting caspase signaling in mice. Am J Nephrol 40: 215-223, 2014.

21. Haylor JL, Harris KP, Nicholson ML, Waller HL, Huang Q and Yang B: Atorvastatin improving renal ischemia reperfusion injury via direct inhibition of active caspase-3 in rats. Exp Biol Med (Maywood) 236: 755-763, 2011.

22. Fisher JW: Erythropoietin: physiology and pharmacology update. Exp Biol Med (Maywood) 228: 1-14, 2003.

23. Pellegrini L, Bennis Y, Velly L, Grandvuillemin I, Pisano P, Bruder N and Guillet B: Erythropoietin protects newborn rat against sevoflurane-induced neurotoxicity. Paediatr Anaesth 24: 749-759, 2014.

24. Liu L, Liu C, Hou L, Lv J, Wu F, Yang X, Ren S, Ji W, Wang M and Chen L: Protection against ischemia/reperfusion-induced renal injury by co treatment with erythropoietin and sodium selenite. Mol Med Rep 12: 7933-7940, 2015.

25. Zhang J, Zou YR, Zhong X, Deng HD, Pu L, Peng K and Wang L: Erythropoietin pretreatment ameliorates renal ischaemia-reperfusion injury by activating PI3K/Akt signalling. Nephrology (Carlton) 20: 266-272, 2015.

26. Kwon MS, Kim MH, Kim SH, Park KD, Yoo SH, Oh IU, Pak S and Seo YJ: Erythropoietin exerts cell protective effect by activating PI3K/Akt and MAPK pathways in C6 cells. Neurol Res 36: 215-223, 2014

27. Li XJ, Zhang GX, Sun N, Sun Y, Yang LZ and Du YJ: Protective effects of erythropoietin on endotoxin-related organ injury in rats. J Huazhong Univ Sci Technolog Med Sci 33: 680-686, 2013.

28. Cugini D, Azzollini N, Gagliardini E, Cassis P, Bertini R, Colotta F, Noris M, Remuzzi G and Benigni A: Inhibition of the chemokine receptor CXCR2 prevents kidney graft function deterioration due to ischemia/reperfusion. Kidney Int 67: 1753-1761, 2005.

29. Zeng Y, Adamson RH, Curry FR and Tarbell JM: Sphingosine-1-phosphate protects endothelial glycocalyx by inhibiting syndecan-1 shedding. Am J Physiol Heart Circ Physiol 306: H363-H372, 2014.

30. Zeng Y, Liu XH, Tarbell J and Fu B: Sphingosine 1-phosphate induced synthesis of glycocalyx on endothelial cells. Exp Cell Res 339: 90-95, 2015.

31. Ratilal BO, Arroja MM, Rocha JP, Fernandes AM, Barateiro AP, Brites DM, Pinto RM, Sepodes BM and Mota-Filipe HD: Neuroprotective effects of erythropoietin pretreatment in a rodent model of transient middle cerebral artery occlusion. $\mathrm{J}$ Neurosurg 121: 55-62, 2014.

32. Jun JH, Jun NH, Shim JK, Shin EJ and Kwak YL: Erythropoietin protects myocardium against ischemia-reperfusion injury under moderate hyperglycemia. Eur J Pharmacol 745: 1-9, 2014.

33. Lu MJ, Chen YS, Huang HS and Ma MC: Erythropoietin alleviates post-ischemic injury of rat hearts by attenuating nitrosative stress. Life Sci 90: 776-784, 2012.

34. Xu X, Cao Z, Cao B, Li J, Guo L, Que L, Ha T, Chen Q, Li C and Li Y: Carbamylated erythropoietin protects the myocardium from acute ischemia/reperfusion injury through a PI3K/Akt-dependent mechanism. Surgery 146: 506-514, 2009.

35. Soranno DE, Rodell CB, Altmann C, Duplantis J, Andres-Hernando A, Burdick JA and Faubel S: Delivery of interleukin-10 via injectable hydrogels improves renal outcomes and reduces systemic inflammation following ischemic acute kidney injury in mice. Am J Physiol Renal Physiol 311: F362-F372, 2016.

36. Sun P, Liu J, Li W, Xu X, Gu X, Li H, Han H, Du C and Wang H: Human endometrial regenerative cells attenuate renal ischemia reperfusion injury in mice. J Transl Med 14: 28, 2016.

37. Segawa R, Mizuno N, Hatayama T, Jiangxu D, Hiratsuka M, Endo $\mathrm{Y}$ and Hirasawa N: Lipopolysaccharide-activated leukocytes enhance thymic stromal lymphopoietin production in a mouse air-pouch-type inflammation model. Inflammation 39: 1527-1537, 2016

38. Takai T: TSLP expression: cellular sources, triggers, and regulatory mechanisms. Allergol Int 61: 3-17, 2012.

This work is licensed under a Creative Commons Attribution-NonCommercial-NoDerivatives 4.0 International (CC BY-NC-ND 4.0) License. 